\title{
Evaluación externa de los aprendizajes
}

Emilio Pedrinaci et al.

Alambique., núm. 37., Didáctica de las Ciencias Experimentales. Julio-Agosto-Septiembre. 2003. Editorial Graó., Barcelona., 126 págs.

\section{DATOS SOBRE LOS COLABORADORES}

Emilio Pedrinaci (Consejo de Dirección de Alambique). News Sanmarti (Departamento de Didáctica de la Matemática y de las Ciencias Experimentales en la Universidad Autónoma de Barcelona). Vicente Doménech (Inspección Educativa Castellón de la Plana). Raimundo Rubio (Instituto Vasco de Evaluación e Investigación en Bilbao). Juan Nieda (Inspección de Educación de Madrid). Ana Cañas (Instituto Julio Palacios, San Sebastián de los Reyes, Madrid). Ma. Jesús Martín-Díaz (Instituto Antonio Machado, Madrid). Conxita Mayós (Organización Curricular del Departamento de Enseñanza de la Generalidad de Cataluña). Ma. del Pilar Menoyo (Instituto J.M. Zafra de Barcelona). Luis Rebollo (Universidad de Alcalá, Alcalá de Henares, Madrid). Juana Nieda (Inspección de Educación, Madrid). Juan O. Centeno, Álvaro García Quintana y Fermín Villarroya (Universidad Complutense de Madrid). Javier González Díez (Instituto Alpedrete de Madrid). José Ma. Pastor (Instituto Pérez Galdós de Madrid). Manel Belmonte (Instituto Narcis Monturiol de Barcelona). Pedro Membiela (Departamento de Didáctica de las Ciencias Experimentales, Facultad de Ciencias de la Educación, Universidad de Vigo, Orense). Ángel de Andrea González (Instituto Alpajés en Aranjuez, Madrid). Ana Gómez Gómez (Instituto San Isidro de Madrid). Bernat Martínez Sebastián (Benidorm, Alicante). Ángel Juan Martínez y Mercedes Juliá Espí (Instituto Pare 
Arques de Cocentaina). Juan Maldonado Román (Instituto Cerro del Viento, Benalmádena, Málaga). Pedro Ramírez Moreno (Instituto Carlinda, Málaga). Fernando Enríquez Enríquez (Facultad de Ciencias de la Universidad de Málaga).

\section{CONTENIDO}

La presentación de la revista está a cargo de Emilio Pedrinaci, consejero de la misma. Incluye cuatro secciones: Monografía, Intercambio, Intercambio-Notas e Informaciones.

Monografía comprende ocho artículos:

1. Evaluación externa: ¿por qué y para quée, de News Sanmarti. Fundamenta la evaluación externa y señala beneficios y problemas.

2. El Proyecto PISA. Un proyecto internacional para la búsqueda de indicadores de rendimiento, de Vicente Doménech. Presenta el organigrama del proyecto y explica sus fases. Muestra resultados de la aplicación en estudiantes de Ciencias.

3. El proyecto TIMSS: evaluación de las ciencias, de Raimundo Rubio. Presenta los resultados de esta prueba internacional, aplicada a estudiantes de Ciencias, y los relaciona con el currículo y el contexto.

4. La evaluación de Ciencias de la Naturaleza en la educación secundaria obligatoria, de Juana Nieda, Ana Cañas y Ma. de Jesús Martín-Díaz. El estudio lo realizan con alumnos de 12, 14 y 16 años.

5. Evaluación de competencias básicas en Cataluña, de Conxita Mayos. Define las competencias y evalúa competencias lingüísticas, matemáticas y socio-científicas.

6. Evaluación de habilidades científicas en las PAAU de Biología, de Ma. del Pilar Menoyo. Categoriza la muestra estudiada, indica las posibles influencias en los resultados de las pruebas y la influencia de la corrección en los resultados de la prueba. 
7. Análisis de la pruebas de acceso a la Universidad de la asignatura de Ciencias de la Tierra y del Medio Ambiente, de Luis Rebollo, Juana Nieda, Juan D. Centeno, Álvaro García Quintana, Javier González Díez y Fermín Villarroya. Analizan la pertinencia de la evaluación de los conocimientos de esta nueva asignatura para la admisión a la Universidad.

8. La Olimpiada de Física, de José Ma. Pastor. Investiga el nivel de preparación de los alumnos que desean concursar en esta prueba.

Intercambio abarca cuatro artículos:

a) Los trabajos de investigación: una innovación en el currículo de bachillerato de Cataluña, de Manel Belmonte. Muestra las aportaciones a la formación de los alumnos, la organización de los trabajos y el tipo de investigaciones realizadas.

b) Dos proyectos curriculares innovadores para la enseñanza de las Ciencias orientada a la relevancia social y personal, de Pedro Membiela. Analiza la influencia de aspectos científicos en el desarrollo de los alumnos a nivel personal y social.

c) Una experiencia sencilla de Física en el aula y su utilidad en el aprendizaje alternativo, de Ángel de Andrea González y Ana Gómez Gómez. Investigan la aplicación de Física y Química en experiencias sencillas en el aula para medir la adquisición de destrezas.

d) Introducción del concepto de aceleración, utilizando programas informáticos de simulación, de Bernat Martínez Sebastián y Ángel Juan Martínez y Mercedes Juliá Espi. Muestran la dificultad de los estudiantes para captar el concepto y cómo lo adquieren con más facilidad por medio de la simulación.

Intercambio-Notas comprende un solo artículo:

El ruido de los ciclomotores como fuente de contaminación, de Juan Maldonado Román, Pedro Ramírez Moreno y 
Fernando Enríquez Enríquez. Proponen contenidos transversales para lograr un cambio de actitud y reducir el ruido.

En Informaciones se participa la noticia de la formación del "Grupo Mujeres en Física»; se presentan los conocimientos más relevantes en tres jornadas celebradas en diferentes sitios; y se reseñan dos libros.

\section{VALORACIÓN CRÍTICA}

Estéticamente, la revista es sombría pues los recuadros están en negro y blanco, muchos con fondo gris. El diseño de las páginas podría ser más agradable.

Los artículos de la sección de Monografía presentan una panorámica sobre diversas evaluaciones, respecto al aprendizaje de las Ciencias en nivel de secundaria y de bachillerato; explican la prueba PISA propuesta por la Organización para la Cooperación y el Desarrollo Económico (OCDE) y la prueba TIMSS; la evaluación para el ingreso a la Universidad; y otro estudio para investigar el nivel de estudio de alumnos que desean competir en la Olimpiada de Física.

Los cuatro artículos comprendidos en Intercambio, investigan la capacidad desarrollada en los alumnos para elaborar ciencia, para mejorar la enseñanza y dos experimentos: uno para optimizar la enseñanza de la Física y el otro de aprendizaje por simulación.

En Intercambio-Notas plantean un proyecto para reducir la contaminación por ruido.

Ana Teresa López de Llergo Villagómez 